N. E. Piskunov, W. W. Weiss, D. F. Gray, eds.

\title{
Simulation of Solar Magnetoconvection
}

\author{
A. Vögler, S. Shelyag, M. Schüssler \\ Max-Planck-Institut für Aeronomie, Max-Planck-Strasse 2, 37191 \\ Katlenburg-Lindau, Germany \\ F. Cattaneo, T. Emonet, T. Linde \\ University of Chicago, Astronomy and Astrophysics, ASCI Flash \\ Center, 5640 South Ellis Ave., Chicago, IL 60637
}

\begin{abstract}
We present a new 3D MHD code for the simulation of solar magnetoconvection. The code is designed for use on parallel computers and in the choice of methods emphasis has been laid on efficient parallelization. We give a description of the numerical methods and discuss the non-local and non-grey treatment of the radiative transfer. Test calculations underlining the importance of non-grey effects and first results of the simulation of a solar plage region are shown.
\end{abstract}

\section{Introduction}

The interaction between convective motions and magnetic fields in the solar photosphere and in the uppermost layers of the convection zone plays an important role for many observable phenomena of solar activity and for the large heating of the upper atmospheric layers. Since any observation of the sun can only give a two dimensional map of quantities integrated along the line of sight, and since sub-photospheric layers are not amenable to direct observation, numerical simulations are necessary to obtain a full three dimensional picture of the relevant physical processes.

Furthermore, the hydrodynamic and magnetic Reynolds numbers of the convective flows result in the formation of magnetic structures with scales much smaller than the resolution limit of presently used telescopes, so simulations provide a way to gather information about the physics of these otherwise inaccessible scales. Realistic simulations of solar magnetoconvection require a detailed non-local and non-grey radiative transfer and need to include compressibility and the effects of partial ionization. The large range of spatial scales to be covered calls for simulation codes designed for use on parallel computers. We have developed a 3D MHD code for applications in the solar photosphere and convection zone that meets these requirements. The choice of a domain decomposition parallelization model in combination with a short characteristics solver for the radiative transfer leads to efficient parallelization, especially of the time consuming radiative transfer calculations. In section 2 . we describe the code and discuss methods and tests of the non-grey radiative transfer. In section 3 . we 
present first results of the simulation of a solar plage region obtained with the code.

\section{Equations and numerical methods}

\subsection{Basic equations and integration scheme}

We set out from the MHD equations consisting of the continuity equation,

$$
\frac{\partial \rho}{\partial t}+\nabla \cdot(\rho \mathbf{u})=0
$$

the equation of motion,

$$
\frac{\partial \rho \mathbf{u}}{\partial t}+\nabla \cdot\left(\rho \mathbf{u u}+\left(p+\frac{|\mathbf{B}|^{2}}{8 \pi}\right) \underline{\mathbf{1}}-\frac{\mathbf{B B}}{4 \pi}\right)=\rho \mathbf{g}+\nabla \cdot \underline{\underline{\tau}},
$$

the energy equation,

$$
\begin{aligned}
\frac{\partial e}{\partial t} & +\nabla \cdot\left(\mathbf{u}\left(e+p+\frac{|\mathbf{B}|^{2}}{8 \pi}\right)-\frac{1}{4 \pi} \mathbf{B}(\mathbf{u} \cdot \mathbf{B})\right) \\
& =\frac{1}{4 \pi} \nabla \cdot(\mathbf{B} \times \eta \nabla \times \mathbf{B})+\nabla \cdot(\mathbf{u} \cdot \underline{\underline{\tau}})+\nabla \cdot\left(\chi \rho \nabla \frac{e}{\rho}\right) \\
& +\rho(\mathbf{g} \cdot \mathbf{u})+Q_{r a d},
\end{aligned}
$$

and the induction equation,

$$
\frac{\partial \mathbf{B}}{\partial t}+\nabla \cdot(\mathbf{u B}-\mathbf{B u})=-\nabla \times(\eta \nabla \times \mathbf{B}) .
$$

Here $\rho$ is the mass density, $\mathbf{u}$ the flow velocity, $\mathbf{B}$ the magnetic field and $e=e_{\text {int }}+e_{\text {kin }}+e_{\text {mag }}$ the total energy density per volume, i.e., the sum of internal, kinetic and magnetic energy densities. $p$ is the gas pressure, $g$ the vector of gravitational acceleration, and $Q_{r a d}$ the radiative source term which is discussed in detail in Sec. 2.3., $\eta$ is the (constant) magnetic diffusivity, $\underline{\underline{\tau}}$ the viscous stress tensor and $\chi$ the thermal diffusivity. The system of equations (1)(4) is solved on a three-dimensional cartesian grid. All spatial derivatives except those involving $\underline{\underline{\tau}}$ and $\chi$ are discretized using 4 th order centered differences on a $5^{3}$ point stencil. Typical values for the Reynolds number in the solar photosphere and upper convection zone lead to structures much smaller than any affordable grid resolution. In order to supress unresolved structures that would destabilize the numerical scheme we adopt the methods described by Caunt \& Korpi (2001) and Stein \& Nordlund (1998). The terms involving $\tau$ and $\chi$ are discretized using 2nd order centered differences and contain artificial diffusivities which prevent the build-up of energy at small scales while leaving resolved scales unaffected.

The code is parallelized by means of domain decomposition. The computational domain is decomposed into a three dimensional array of rectangular subdomains, each of which is equipped with two layers of ghost cells at each of its boundaries, as required by the 4th-order scheme. Time stepping is explicit, using a 4th-order Runge-Kutta scheme. The code was tested with a series of standard test problems. 


\subsection{Equation of state}

The equation of state closes the system of equations (1)-(4) by specifying the relations between the thermodynamical quantities of the fluid. In the solar photosphere and upper convection zone, the deviations from the ideal gas law due to partial ionization of the fluid are important since transport of latent heat contributes significantly to the total convective energy transport. We take into account the first ionization of the eleven most abundant element. The internal energy per mass unit $\epsilon=e_{\text {int }} / \rho$ can then be written as

$$
\epsilon=\frac{3}{2 \rho}\left(n_{e}+n_{a}\right) k T+\frac{1}{\rho} \sum n_{i}^{*} \chi_{i},
$$

where the sum runs over the particle species (labeled with index $i$ ), $n_{i}^{*}$ is the number density of ionized particles of type $i$, and $\chi_{i}$ the corresponding ionization energy. $n_{a}=\sum n_{i}$ is the number density of nuclei, $n_{e}$ the number density of electrons. Defining the ionization degree, $x_{i}=n_{i}^{*} / n_{i}$, and the relative abundance, $\nu_{i}=n_{i} / n_{a}$, Eq. (5) can be rewritten as

$$
\epsilon=\frac{3 k T}{2 \mu_{a} m_{0}}\left(1+\sum x_{i} \nu_{i}\right)+\frac{1}{\mu_{a} m_{0}} \sum x_{i} \nu_{i} \chi_{i},
$$

where $\mu_{a}=1.29$ is the mean molecular weight of the neutral gas and $m_{0}$ is the atomic mass unit. The ionization degrees, $x_{i}$, are determined by the set of Saha equations

$$
\frac{x_{i}}{1-x_{i}} \sum x_{i} \nu_{i}=\frac{u_{i 1}(T)}{u_{i 0}(T)} \frac{\mu_{a} m_{0}}{\rho} \frac{2\left(2 \pi m_{e} k T\right)^{3 / 2}}{h^{3}} \exp \left(-\chi_{i} / k T\right) .
$$

$\mathrm{Up}$ to a temperature of $16000 \mathrm{~K}$ the temperature dependence of the partition functions $u_{i 1}, u_{i 0}$ was taken from Irwin (1981), for higher temperatures the elements considered are almost fully ionized and the temperature dependence can be neglected. In order to obtain the temperature, the nonlinear system of equations (6) and (7) is solved iteratively. Once the temperature is known, the gas pressure follows from

$$
p=\left(n_{e}+n_{a}\right) k T=\frac{\rho}{\mu_{a} m_{0}}\left(1+\sum x_{i} \nu_{i}\right) k T .
$$

The equation of state is stored in tables from which the required quantities are interpolated during a simulation run.

\subsection{Radiative transfer}

The radiative source term in Eq. (3) is written as

$$
Q_{\text {rad }}=-\int_{\nu}\left(\nabla \cdot \mathbf{F}_{\nu}\right) d \nu=4 \pi \rho \int_{\nu}\left(J_{\nu}-S_{\nu}\right) d \nu
$$

The numerical evaluation requires either the radiation flux vector, $\mathbf{F}_{\nu}=$ $\int_{\nu} \mathrm{I}_{\nu}(\underline{\omega}) \underline{\omega} \mathrm{d} \omega$, or the average intensity, $J_{\nu}=\frac{1}{4 \pi} \int I_{\nu}(\underline{\omega}) d \omega$, to be calculated. Either of the quantities can be determined by solving the radiative transfer equation,

$$
\frac{d I_{\nu}}{d s}=-\kappa_{\nu} \rho\left(I_{\nu}-S_{\nu}\right),
$$


for the specific intensity $I_{\nu}$ along rays for a number of directions and frequencies $\left(\kappa_{\nu}\right.$ is the opacity and $S_{\nu}=B_{\nu}$ for LTE). In order to keep the radiative transfer numerically tractable, the frequency dependence must be accounted for in a statistical way. The simplest and most time-efficient approach would be the grey approximation. While it can be expected to describe the interaction between convective motions and radiative cooling in the layer around continuum optical depth unity with sufficient accuracy, it neglects the effects of line cooling and backwarming, which have a strong influence on the temperature structure of the upper photosphere. As we shall show below, the errors of the grey approximation become particularly pronounced in situations strongly deviating from a plane-parallel atmosphere, where lateral heating effects come into play. Such situations are typically encountered in simulations of magnetoconvection. Following Nordlund (1982), Ludwig (1992) and Ludwig et al. (1994)

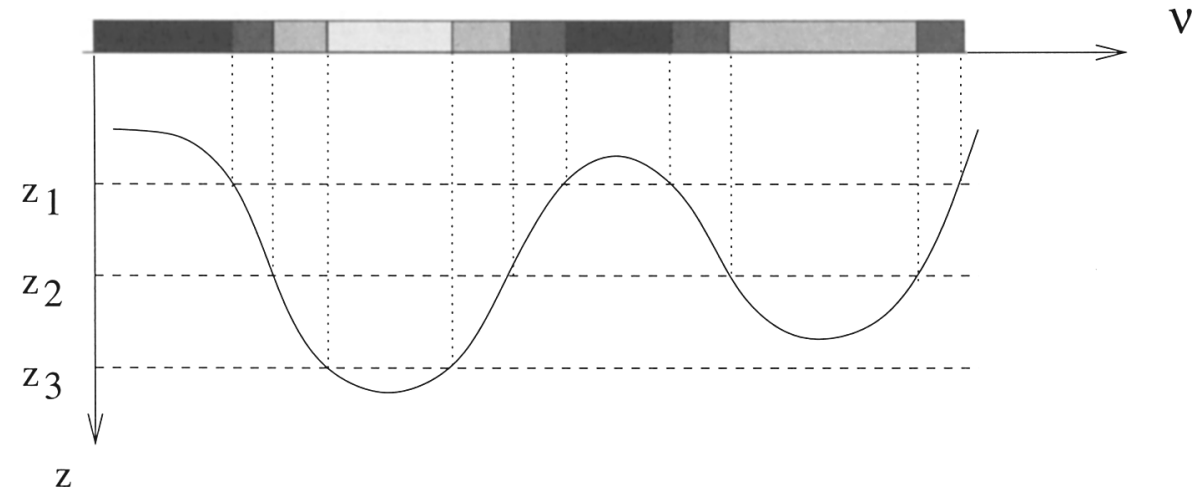

Figure 1. Illustration of the frequency sorting scheme. The solid curve indicates the height in a reference atmosphere where $\tau_{\nu}=1$ is reached. Frequencies which reach $\tau_{\nu}=1$ in the same height interval $\left(z_{i}, z_{i+1}\right)$ are binned into one frequency set.

we take into account the non-grey character of the radiative transfer by using the 'opacity binning'. The basic idea is to sort frequencies into sets (typically $4-6$ ) according to the geometrical depth in a 1D reference atmosphere at which optical depth $\tau_{\nu}=1$ is reached (see Fig. 1). Then for each frequency set $i$ a transfer equation

$$
\frac{d I_{i}}{d s}=-\bar{\kappa}_{i} \rho\left(I_{i}-\bar{B}_{i}\right)
$$

with a set-integrated source function, $\bar{B}_{i}$, and a set-averaged opacity, $\bar{\kappa}_{i}$, is solved. We use the Rosseland mean (taken over the frequency set) in optically thick regions and make a smooth transition to the Planck mean in optically thin layers, based on an estimate of the local optical depth given by $\tau_{i} \approx \bar{\kappa}_{i, R o s} p / g$.

For the angular discretization we use the quadrature formulae of set $\mathrm{A}$ by Carlson (1963). At a given grid point, the radiative flux and average intensity are then calculated as (dropping the set index) $F_{j}=4 \pi \sum_{l} w_{l} \mu_{j l} I_{l} \quad(j=x, z, y)$ and $J=\sum_{l} w_{l} I_{l}$, respectively, where the sum runs over the ray directions. The 
coefficients $w_{l}$ are the weights normalized to unity and $\mu_{j l}$ is the direction cosine. For the calculations presented in Sec. 3. the A4 quadrature set with 3 directions per octant was used.

We solve the radiative transfer equation (11) for each frequency set and each direction using the short characteristics scheme as described by Kunasz \& Auer (1988). It is based on the formal solution of the transfer equation, here stated for a given cosine $\mu$ of ray inclination, along the ray segment between neighbouring height levels $i$ and $i+1$

$$
I_{i+1}=I_{i} e^{-\Delta \tau_{i} / \mu}+\frac{1}{\mu} \int_{\tau_{i+1}}^{\tau_{i}} B(\tau) e^{-\left(t-\tau_{i+1}\right) / \mu} d \tau
$$

In order to evaluate $\Delta \tau_{i}=\tau_{i}-\tau_{i+1}$ and the integral contribution to Eq. (12), the values of $\kappa, \rho$ and $B$ are interpolated linearly along the ray segment. This results in polynomial expressions, which can be evaluated analytically; alternatively a parabolic interpolation can be used for $\kappa$ and $B$. In plane-parallel calculations, the upwind intensity $I_{i}$ has been obtained in the previous step of the computation. In $2 \mathrm{D}$ and $3 \mathrm{D}$, the ray is traced back from point $i+1$ until it reaches a cell boundary. At the point of intersection, the upwind intensity $I_{i}$ is interpolated from the values at the neighbouring grid points. In $2 \mathrm{D}$, linear or parabolic interpolation can be used; for 3D calculations we use bilinear interpolation.

In the context of the domain decomposition used by the code, the short characteristics scheme requires an iteration for each ray direction and each frequency set: for a given ray direction the scheme starts in each subdomain at those boundaries through which the radiation flows in (the 'upwind' boundaries). The intensity values at these boundaries are assumed to be known. Then the traversal of the subdomain proceeds in the downwind direction, systematically moving away from the upwind boundaries, thus making sure that the upwind intensities required for the interpolation are always known. However, on those upwind boundaries of a subdomian which do not coincide with the top or bottom boundary of the computational box, the intensities are a priori unknown. Therefore, the scheme is iterated until convergence at the boundaries is obtained. After each iteration the intensities at a given upwind boundary are updated with the new values provided by the neighbouring subdomain. We found that $2-3$ iteration steps per set and direction are usually sufficient, if one chooses as initial guess for the intensities on the upwind boundaries a linear extrapolation of the values of the previous two time steps.

As an example for the importance of non-grey effects and a test of the opacity binning method, we consider a simple 2D magnetic flux sheet model. Using a 1D reference atmosphere (Maltby et al. 1986), the stratification in the interior of the sheet is shifted $200 \mathrm{~km}$ downwards relative to the surrounding. Consequently the sheet is cooler and less dense than the outside atmosphere at the same geometrical height level. The width of the sheet as a function of height results from flux conservation together with the condition of total pressure balance at the interface. At the height $z=0$, corresponding to continuum optical depth unity outside the sheet, a width of $150 \mathrm{~km}$ has been specified. The sheet fans out with increasing height as the magnetic pressure necessary to balance the jump in gas pressure decreases. At the sheet boundaries the transition is smeared out 


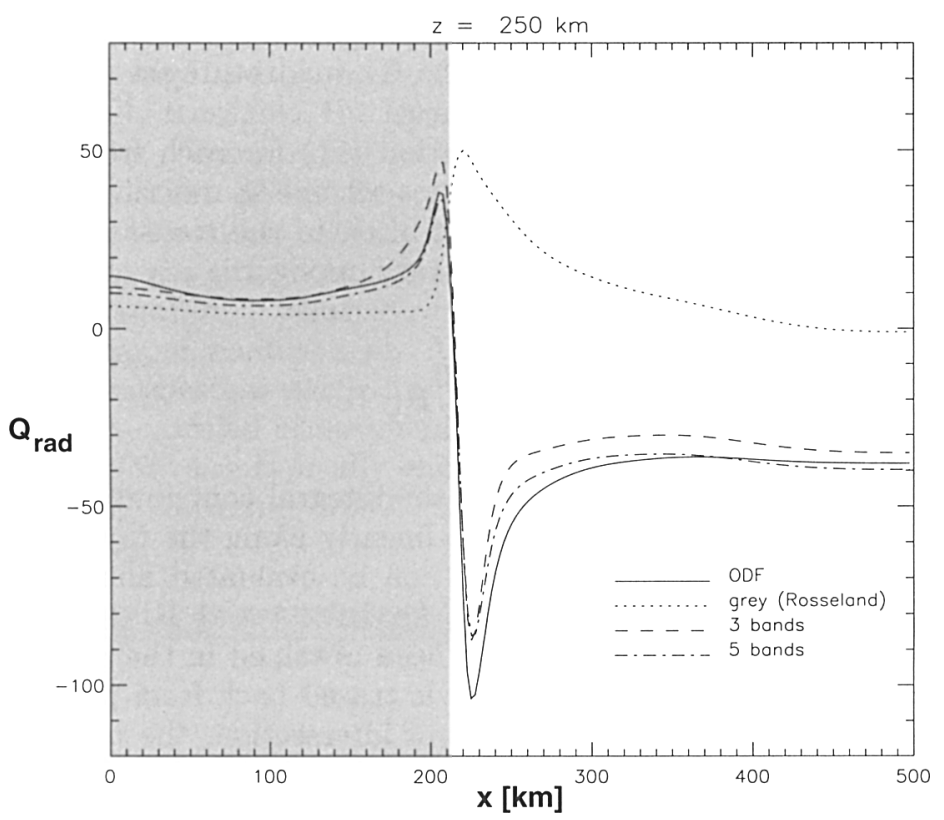

Figure 2. Horizontal cut of $Q_{\text {rad }}$ at a height of $250 \mathrm{~km}$ through the $2 \mathrm{D}$ atmosphere with embedded flux sheet. $x=0$ corresponds to the symmetry axis of the sheet. The sheet interior is shaded in grey. The opacity binning models yield a much better approximation of the lateral heating and cooling near the interface than the grey solution.

horizontally over a distance of $40 \mathrm{~km}$. Fig. 2 shows $Q_{\text {rad }}$ for a horizontal cut through the sheet at height of $250 \mathrm{~km}$ starting from the symmetry axis at $x=0$. Results for the grey (Rosseland) mean and for opacity binning models using 3 and 5 sets are shown. The reference solution was obtain with opacity distribution functions (ODFs) by Kurucz (1979). The 3- and 5-set solutions approximate the 'reference' solution rather well. Both reproduce the characteristic heating peak on the inner side and stronger cooling peak outside the sheet boundary, the 5-set model performing slightly better. The grey solution, however, does not even capture the qualitative behaviour of the reference. The heating peak inside the sheet is missing; instead, heating takes place immediately outside the sheet, where the correct solution shows considerable cooling. The reason for the failure of the grey solution lies in the fact that at a height of $250 \mathrm{~km}$ the interior of the sheet is largely transparent when the Rosseland mean opacity is used. As a result, the interaction between gas and radiation inside the sheet is very weak and the values of $Q_{\text {rad }}$ are accordingly small. In the optically denser regions immediately outside the sheet, radiation which originates from deeper, hotter regions at the opposite sheet boundary and which crosses the sheet almost unattenuated, leads to a net heating. In the opacity binning models, on the other hand, the sheet is still opaque for those frequency sets which represent larger opacity (spectral lines). The situation is illustrated schematically in Fig. 3. The 


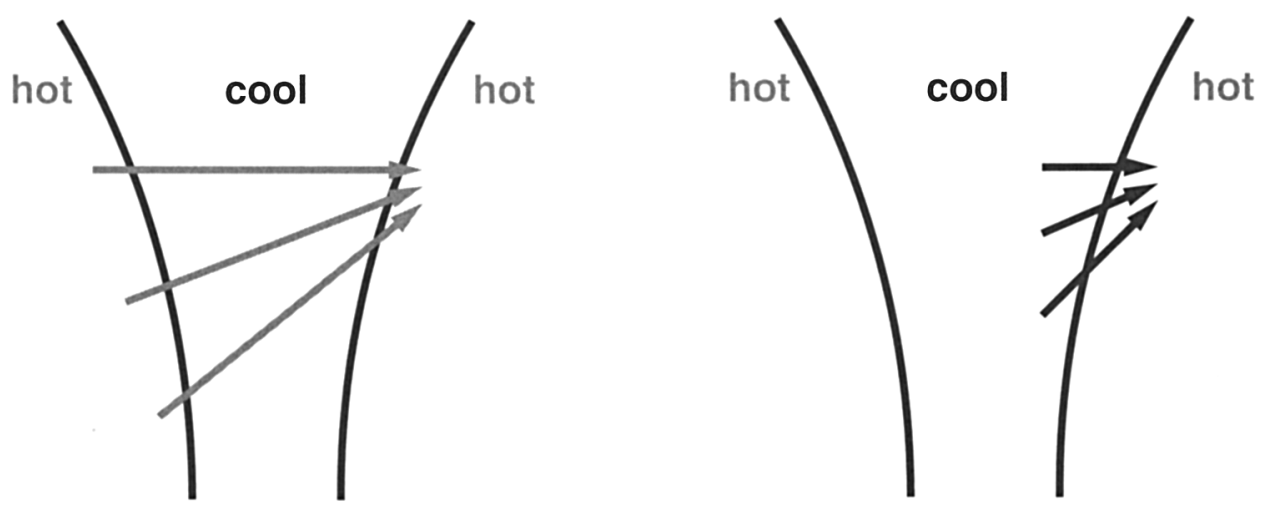

Figure 3. In the grey approximation (left) the upper parts of the flux sheet are almost transparent. At the same height, the sheet still appears opaque for opacity binning models (right).

error of the grey solution far outside the sheet $(x=500 \mathrm{~km})$ is in accordance with the results of $1 \mathrm{D}$ calculations in the plane-parallel reference atmosphere atmosphere.

\section{First results}

In this section we show some results of a first simulation run with the new code. The dimensions of the computational domain are $1400 \mathrm{~km}$ in the vertical direction and $6000 \mathrm{~km}$ in both horizontal directions, with a resolution of $100 \times 288 \times 288$ grid points. The top and bottom boundaries of the domain are closed with stress free boundary conditions for the horizontal velocity components; the implementation of more realistic transmitting upper and open lower boundaries is under development. The magnetic field is assumed to be vertical at the top and bottom boundaries, the footpoints of field lines are allowed to move freely. The horizontal directions are taken to be periodic. In this first run we have used the grey Rosseland approximation for the radiative transfer. The simulation starts with a plane-parallel standard solar atmosphere (Spruit 1974) extending from $800 \mathrm{~km}$ below to $600 \mathrm{~km}$ above the level of continuum optical depth unity. After convection has fully developed, a upward-directed homogeneous magnetic field of $200 \mathrm{G}$ is introduced. Within a few minutes of simulated time (approximately one turnover-time of the convection) most of the magnetic flux has been transported to the downflow lanes of the convection (granulation) pattern. Fig. 4 shows a horizontal cut at the $\langle\tau\rangle=1$ level several turnover times after the start of the magnetic phase. Thin sheet-like magnetic structures extend along intergranular downflow lanes, while larger structures with diameters of $800-1000 \mathrm{~km}$ (comparable to small pores on the sun) are formed at granule vertices where several downflow lanes merge. Typical field strengths in these field concentrations at a height corresponding to optical depth unity are between 1700 and $2000 \mathrm{G}$. In the intensity picture, the 'mini-pores' appear dark owing to the reduced efficiency of convective energy transport and hence lower 

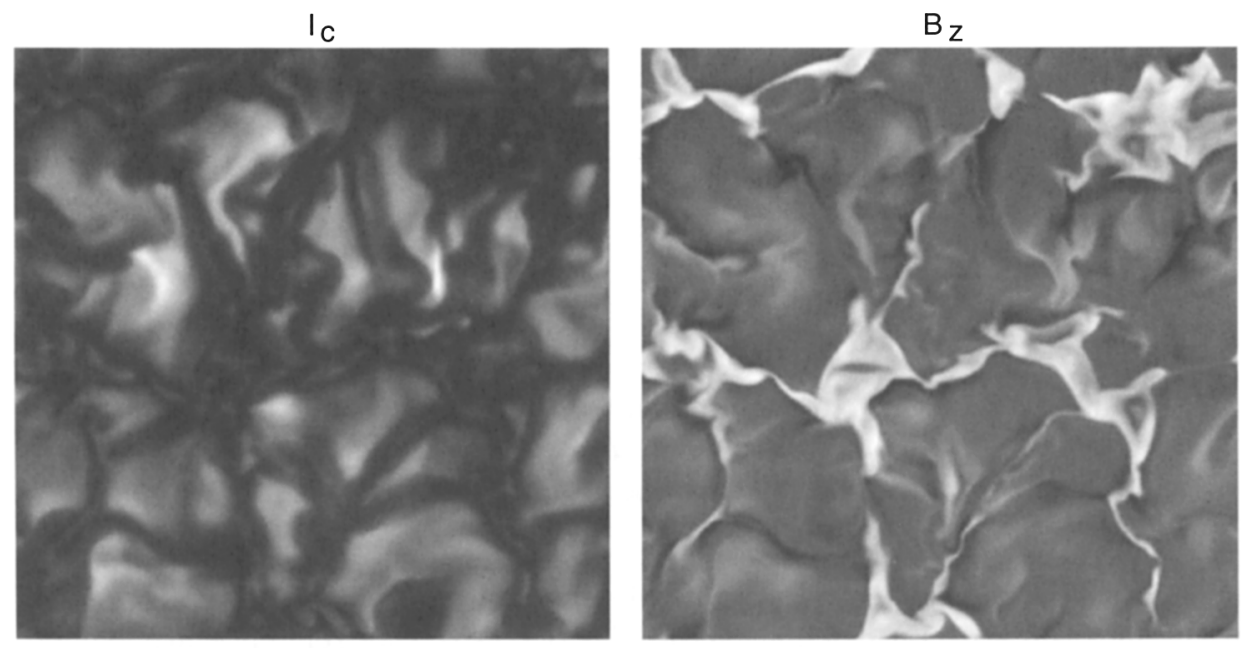

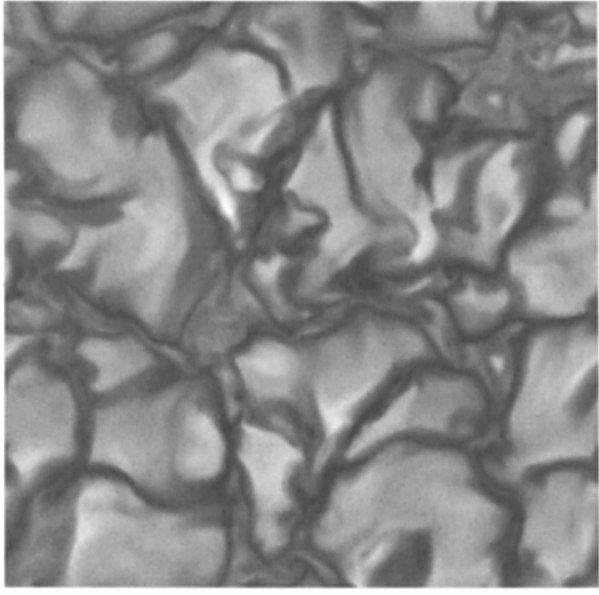

$v_{Z}$

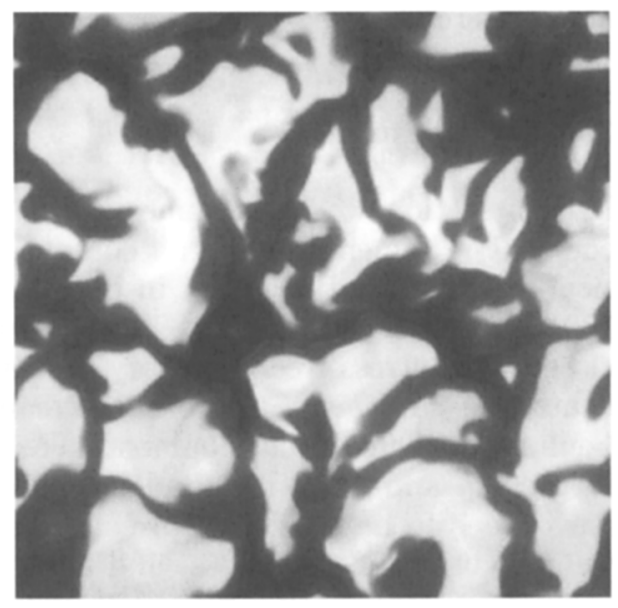

T

Figure 4. Intensity picture and horizontal cuts at $\langle\tau\rangle=1$ of vertical magnetic field, temperature and vertical velocity (clockwise, from top left). Light and dark shades indicate higher and lower values, respectively. The $v_{z}$ plot shows granular upflows shaded in light grey separated by intergranular downflow lanes shown in dark shades. In the $B_{z}$ plot, the 'meso-scale' network of strong sheet- and pore-like magnetic field concentrations is shown in white. 

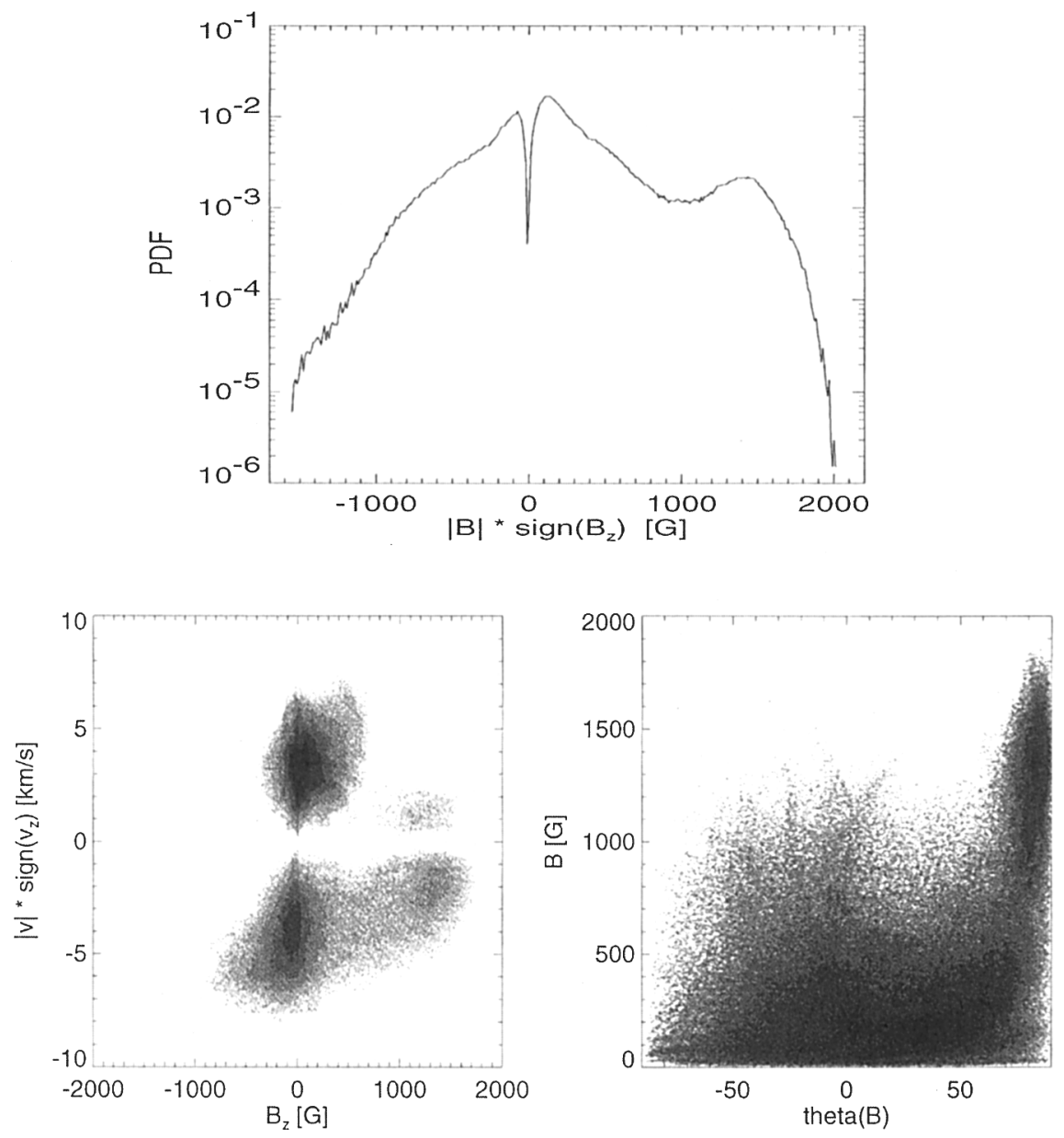

Figure 5. Statistical properties of a layer of $100 \mathrm{~km}$ thickness around $\langle\tau\rangle=1$. Top panel: Probability distribution function (PDF) for $|\mathbf{B}|$, signed with vertical orientation. Bottom panels: correlation diagrams (joint PDFs); the grey-scaling shows the probability on a logarithmic scale. theta $(B)$ is the inclination angle of $\mathbf{B}$ with respect to the horizontal. 
temperature at optical depth unity. In the thin sheets, lateral heating effects in combination with the depression of the $\langle\tau\rangle=1$ level lead to a brightening with respect to the surrounding downflow regions. The larger magnetic elements reveal internal small-scale structure with bright hot upflows in regions of reduced field strength. The network of magnetic structures is organized on a 'meso-scale' which typically comprises $4-6$ granules. While this magnetic pattern is rather stable (it evolves on a time scale much larger than the granule lifetime), the small-scale pattern of the field concentrations is highly time-dependent, with magnetic flux being constantly redistributed within the magnetic network. Fig. 5 shows some statistical properties of a layer of $100 \mathrm{~km}$ thickness around $\langle\tau\rangle=1$ corresponding to the snapshot shown in Fig. 4. The probability distribution function (PDF) for the field strength (Fig. 5, top panel) reveals two components: a weak-field component with an approximately exponential distribution, occupying most of the volume considered, and a gaussian component of strong upward-directed field with a peak at $1400 \mathrm{G}$, representing the pore- and sheetlike field concentrations. The correlation diagram of magnetic field strength and inclination angle of $\mathbf{B}$ with respect to the horizontal plane (Fig. 5, bottom right panel) shows that almost all the field with a field strength exceeding $1000 \mathrm{G}$ is vertical. The orientation of weak fields is more widely distributed, but a clear trend towards a horizontal orientation can be observed. The bottom-left panel of Fig. 5 shows the influence of strong fields on fluid motions: while up- and downflows with velocities up to $7 \mathrm{~km} / \mathrm{s}$ (the maximum is reached in downflows) can be observed in weak-field regions, the velocity amplitudes are significantly reduced in the presence of strong fields. However, motions are not completely supressed and downflows prevail inside flux concentrations.

\section{Outlook}

The first results of our new code look promising. They are in agreement with the basic picture of solar magnetoconvection obtained by previous simulations, but the closed-box boundary conditions and the grey radiative transfer must be regarded as unsatisfactory over-simplifications. Therefore the next steps of our project will be the development of open vertical boundaries and the use of our implementation of the non-grey radiative transfer.

\section{Discussion}

STEPIEN: Do you identify regions of the strong magnetic field obtained in the simulations with pores?

VÖGLER: Based on the typical size of $800-1000 \mathrm{~km}$ of these structures, one could identify them with micro pores. A detailed comparison with observations remains to be done, however.

TITLE: On the sun the luminosity does not drop in plage? What do you predict? VÖGLER: In principle the net effect of magnetic fields on luminosity should depend on the size distribution of magnetic structures. However, in our simulation we prescribe the total energy transported across the simulated layers, so I cannot make a statement about plage luminosity based on this simulation. 


\section{References}

Carlson, B. G. 1963, in Methods in Comp. Physics, Vol. 1, p. 1, B. Alder \& S. Fernbach (eds.)

Caunt, S. E., \& Korpi, M. J. 2001, A\&A, 369, 706

Irwin, A. W. 1981, ApJS, 45, 621

Kunasz, P. B., \& Auer, L. 1988, J. Quant. Spectr. Radiat. Transfer, 39, 67

Kurucz, R. L. 1979, ApJS, 40, 1

Ludwig, H. G. 1992, PhD Thesis, Universität Kiel

Ludwig, H. G., Jordan, S., \& Steffen, M. 1994, A\&A, 284, 105

Maltby, P., Avrett, E. H., Carlsson, M., Kjeldseth-Moe, O., Kurucz, R. L., \& Loeser, R. 1986, ApJ, 306, 284

Nordlund, A. 1982, A\&A, 107, 1

Spruit H. C. 1974, SoPh, 34, 277

Stein, R. F., \& Nordlund, Å. 1998, ApJ, 499, 914 